\title{
Research on loT-based Manufacturing Plant Design Models and Sensor DATA Middleware with Event-condition-action
}

\author{
Kyunghoon Jang ${ }^{1, a}$, Jaeil Park ${ }^{2, b,{ }^{*}}$ \\ ${ }^{1,2}$ San 5, Woncheon-Dong, YeongTong-Gu, Suwon, Ajou University, Republic of Korea \\ ajkjang27@gmail.com, bjipark@ajou.ac.kr \\ ${ }^{*}$ Corresponding author
}

Keywords: loT, Middle-ware, Smart factory.

\begin{abstract}
By having the ICT technology development, the sensor based IoT technology is having attention. And there are a lot of research activities to strengthen each company's competitiveness applying to each manufacturing sites. Therefore, in this research, we suggest the IoT manufacturing site platform to apply in industrial sites dependably, and form a middle-ware of ECA rule based real time data treatment and define sensor data based factory control model.
\end{abstract}

\section{Introduction}

There has been ICT technology based solutions released to enhance the inventory management effectiveness to process control and continuous improvement after the appearance of MRP (Material Resource Planning). Especially the production site information gathering solutions had a role in collecting various facility information real time and execute factory automation by combination with PLC (Programmable Logic Control). However, for the MES (Manufacturing Execution System), and PoP (Point of Production), the facility subordinate data were just collected and there were lacking of 4M (Man, Machine, Material, Method) data. [1] Also the data collection by hand-operating with keyboard or external input unit made reliability drop because of the errors (Raw-data), by this it makes us use MES function partially, which is a weakness. Also the data that has no reliability can make frequent errors in automatic control aspect, and this directly influences the drop of reliability in facility itself and the product quality. [2] From this, we can consider the appearance of IoT technology as a strong alternative to settle present problem. However, the research trend of IoT is biased in the developing the sensor itself of data analysis. The reference case for real factory applying model is not enough.

In this research, it will suggest production environment adaptive IoT model to collect data and make them. From the variety of system element, it will introduce the first theme which is data middle-ware including rule engine to sensor data treatment and feedback control.

\section{Research Point}

\section{IoT (Internet of Things)}

The IoT's concept is that the individual object has IP address and become online. Their device connects in network and able to communicate and situation awareness based knowledge service is possible. [3] So the purpose of the IoT technology is to bind the world objects into one and provide service to the principal that needs it. The purpose is to provide service to family or facility by the sensor of measurement. The main factors that forms IoT is RFID, and WSN which is sensor based networking technology to connect objects. [4] The low power and small technical development has made the environment that can attach sensor, and by this a lot of field such as health care, smart factory, and home network system industrial product are blending like network + sensor.

\section{Factory Management and IoT}

To deduct best product(y) in manufacturing environment(W), the key factor(x) managing is important. [5] $\mathrm{X}$ factor is the main reason to get optimum result value from the parameter that constitute process. It can be deducted from $4 \mathrm{M}$. The existing process control method thought of 
human situational awareness or experimental judgments ability as standard, but by the LOT based system's sensor role becoming strengthening, the condition of manufacturing site can be grasped accurately by the measured sensor value. By the development of technology in hardware, the sensor module that has accurate accuracy has increased and the sensor spec that can survive in poor environment has increased. But the professional development is needed in the software and control part in the facility that is related with sensor. Because of this, the cost increases and can't correspond flexibly when the work-flow changes. To supplement these weakness, the new auto control model which is based on the sensor has appeared. This have to correspond to the work-flow which has frequent changes, and define the customizing of end-user easily.

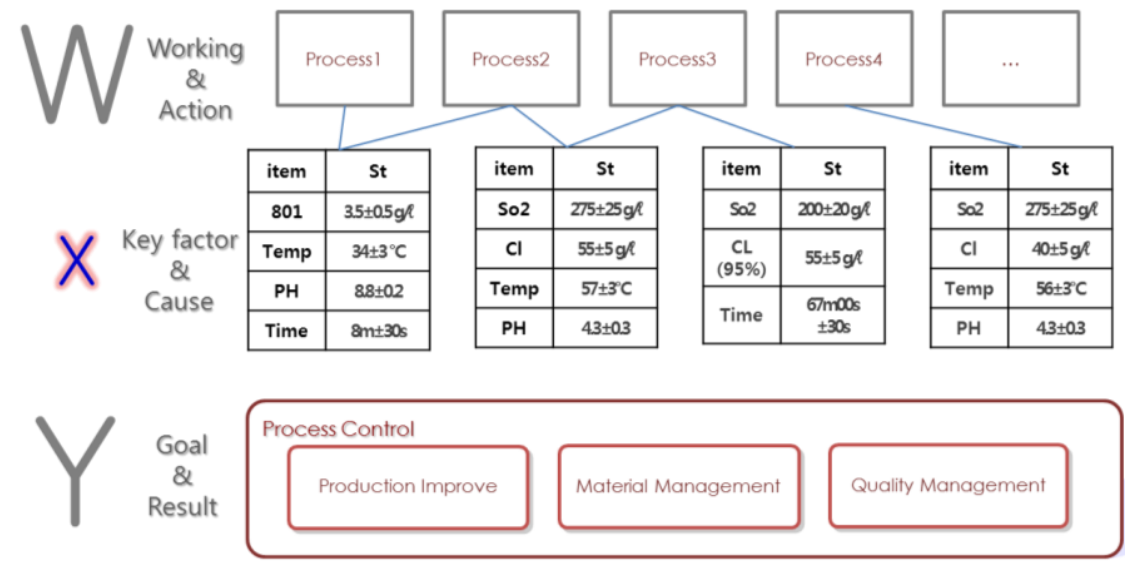

Fig. 1 Process Control AS-IS Model

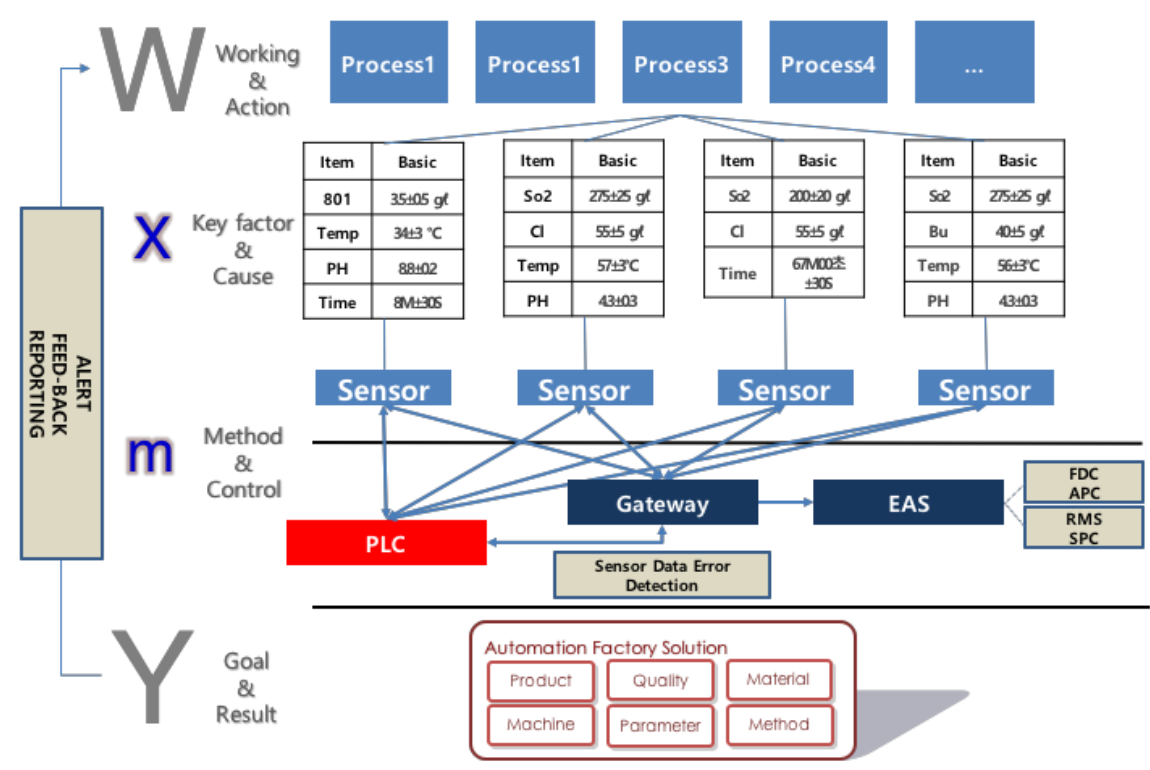

Fig. 2 Process Control To-Be Model

\section{IoT Factory System Model}

Layout of the system for IoT Sensor-based process control configuration as shown in Figure 2. And it contains five categories items.

1. Automation Factory System

$\checkmark \quad$ Main purpose is Data Analysis(Data $\rightarrow$ Information)

$\checkmark \quad$ Information + U.I $\rightarrow$ Monitoring System

$\checkmark \quad$ Error Detection

$\checkmark \quad$ Process Parameter Management

2. Cloud Data Storage and user Interface

$\checkmark \quad$ Stored Data at Cloud

$\checkmark \quad$ Parsing the Data to Mobile or Web Applications

3. IoT Middle-ware 


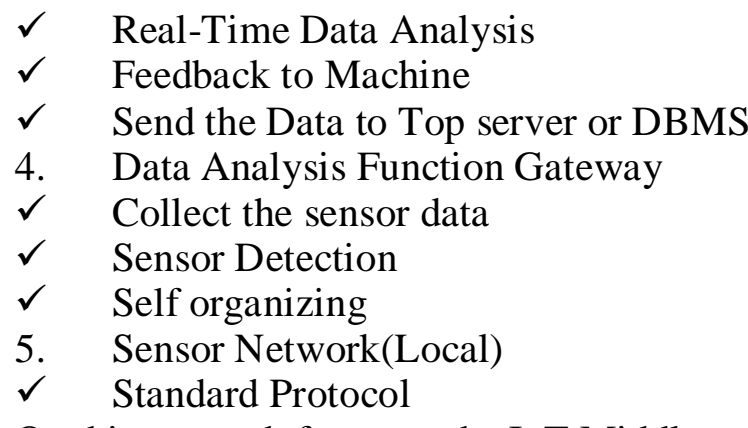

On this research focus on the IoT Middle ware system model with ECA(\#3)

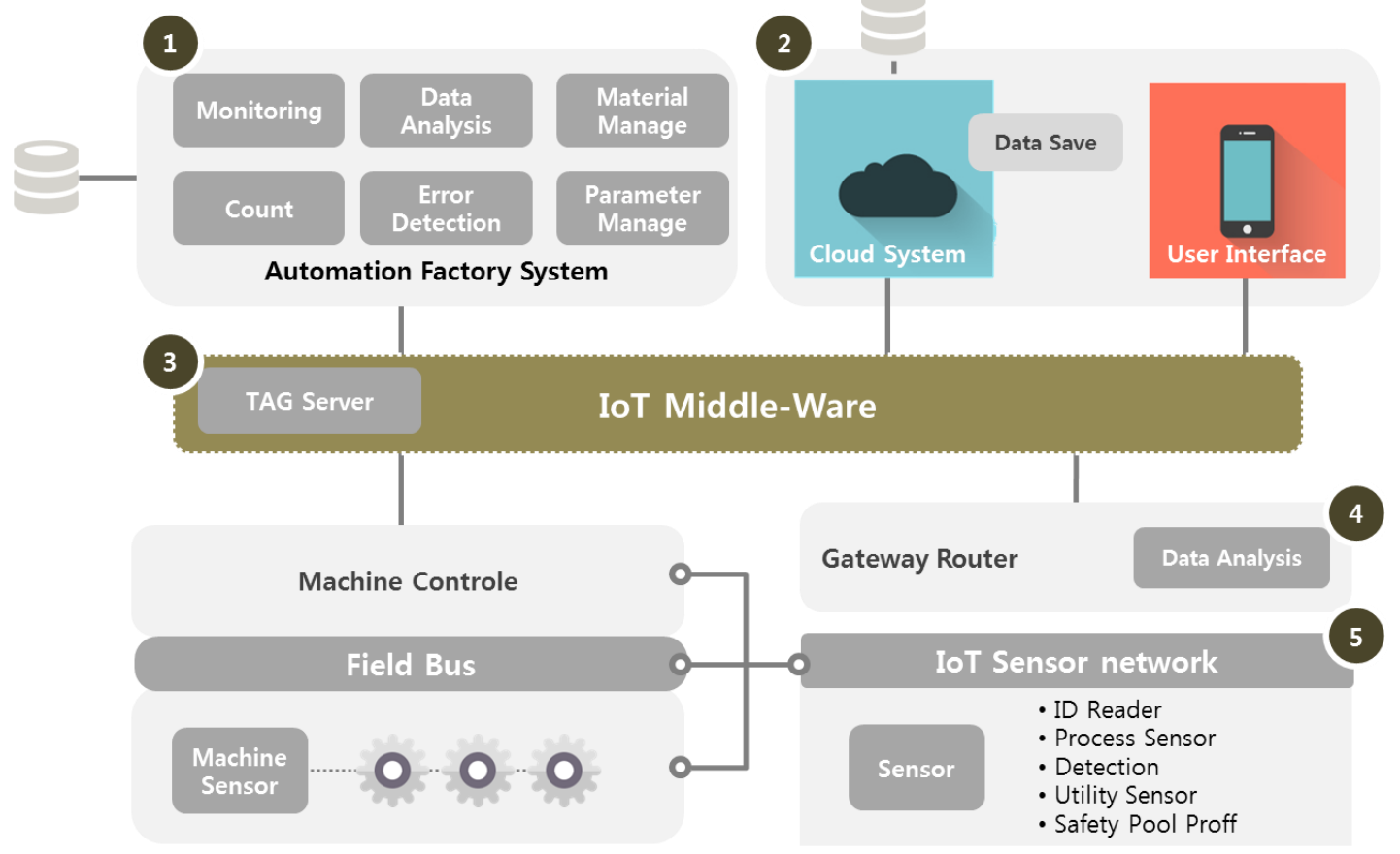

Fig. 3 IoT Factory Control Model

\section{Field type Middle-ware}

The main purpose of field type middle-ware is the handling support of data real time. Because of the characteristic of the manufacturing site which have to get feedback quickly, the delay time of arriving to the outside server and signal coming back more than a second is fatal. To supplement this, there needs to be an engine that determine the urgent data handling. Also because the infra composition has to be possible in the site, heavy solution can cause inconvenience to the network resource so the efficient composition is compulsory. The rule engine which is the main function of middle ware to select data, can sort event situation easily and work-flow connection is possible. This composition is based in the ECA. The ECA basic structure is "On Event If Condition Do Action" and this kind of structure has verified the effect in the activate dispersion database design and semantic application program. It can design efficient event management system in simple structure. [6] Also the ECA rule defines the system design and situation of the incident, and is made to call out the action that is right for that situation. From the existing research, it was used in the code designing plan to increase reliability and development of the ubiquitous area robot. In the G.Knolmayer (2007) [7] thesis, it used ECA rules and defined each work-flow. After defining the process of ARIS, Petri nets, ECA rules, it shows that it can be reproduced to ERP, DBMS, or application code. By referring to the existing research, by the ECA based Rule engine composition, it can be establishing whether to download it into database or not. The database table composition is like table 1.

In the ECA Rule Engine, the value of the sensor that has been modulated are the factor of event, and according to the condition of rule engine, the action is being judged. The final action order is based on ether-cat cable or 802.15 module to have real time control. 


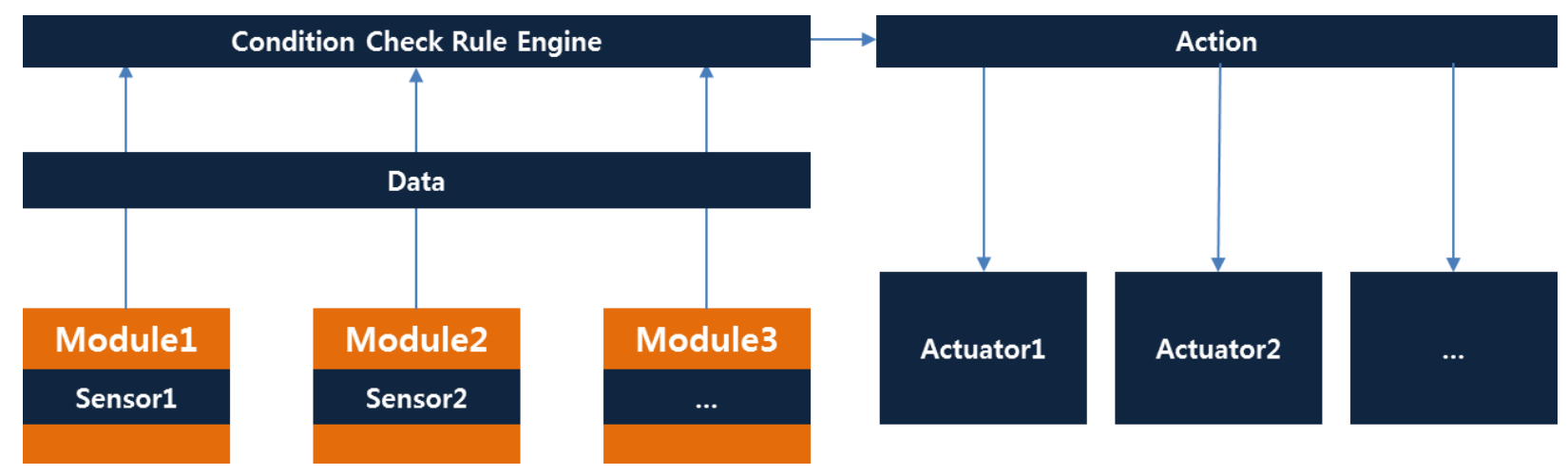

Fig. 4 ECA rule Engine Model

Tab. 1 ECA Database Model

\begin{tabular}{c|c}
\hline Coolum & Description \\
\hline Event_Name & distinguishes Events (Primary Key) \\
\hline Condition_Input & Sensor's Data \\
\hline Condition_Check & Wher Enter the Value for checking the Condition \\
\hline Condition_Location & Target the Action Script Do \\
\hline Action_Location & IP Address for Target \\
\hline Action_Address & Numbering of Action \\
\hline Action_Sequence & Command for Action \\
\hline Action_Excution &
\end{tabular}

\section{Conclusion}

These were the explanation of the middle-ware composition which has ECA rule based to collect data and handle. The logic planning that is based on ECA has an advantage of simple and spread out each situation without exception, able to express complicated work-flow simply. However, to use it in industrial site, we have to think of the verification of communication protocol that is reliable and the real time of synchronization. In the after research, by realizing the model, we need to do supplement and verification and have monitoring system composition and have the additional composition of data analysis model.

\section{References}

[1] S.Y KIM, Study of Digital Factory FOM Solution on Software-based: Applied Case To HeatTreatment Company, Korean Institute of Industrial Engineers, Vol.4, pp.2855-2863, 2015.

[2] S.W Lee J.K Lee S.J Nam J.K Park, Application of Data Acquisition System for MES, The Korean Society of Mechanical Engineers A-35, Vol.9, pp. 1064-1079, 2011.

[3] ITU-T, Overview of the Internet of things, Y.2060, pp. 1-34, Feb. 2012.

[4] J.Gubbi R.Buyya S.Marusic M. Palaniswami. Internet of Things (IoT): A vision, architectural elements, and future directions, Future Generation Computer Systems 29, pp.1645-1660, 2013.

[5] B.J Yum B.Y Lee S.W Ko J.H Kim, Principles for Constructing Taguchi Experimental Designs, The Korean Statistical Society, 1999.

[6] J.W Lee, Development of ECA Rule-Based Complex Event Processing System, Gyeong-Sang National Univ. Master Graduate Paper, 2013.

[7] G.Knolmayer, R.Endl, M.Pfahrer, "Modeling Processes and workflows by business Rules", Lecture Notes in Computer Science, Volume 1806, pp. 16-29, 2000. 